\title{
Effects of Combined Program of Laughter and Exercise vs Exercise- Only Program in Pneumoconiosis Patients
}

\author{
Sungyeau Choi ${ }^{1}$ and Hyunju Park ${ }^{2}$ \\ ${ }^{1}$ Health Care Research Center, Kangwon National University, Korea \\ ${ }^{2}$ School of Nursing, Kangwon National University, Korea \\ ${ }^{1}$ sungyeau@hanmail.net, ${ }^{2}$ hpark@kangwon.ac.kr
}

\begin{abstract}
Previous studies have focused on physical health, but mental health issues such as depression and anxiety are also important in pneumoconiosis patients. Therefore, this study aimed to compare the effects of a combined program of laughter and exercise and an exercise-only program on the physical and mental health of patients with pneumoconiosis. The intervention was conducted with 20 participants in each of the experimental and comparison groups, three times a week for five weeks. The experimental group received a combined intervention of laughter and exercise, and the comparison group received the exercise-only intervention. In the results, no significant difference in physical health variables between the two groups, but within-group pre- and post-data comparison showed that peripheral capillary oxygen saturation $\left(\mathrm{SPO}_{2}\right)$ was significantly increased in the experimental group, and subjectively perceived dyspnea was significantly reduced in both groups. As for the mental health variables, no significant difference in depression was found, while anxiety and quality of life were significantly improved in the experimental group. Thus, the combined program of laughter and exercise had a significant effect on mental health and a partial effect on physical health in patients with pneumoconiosis.
\end{abstract}

Keywords: Laughter, Exercise, Pneumoconiosis, Physical health, Psychological health

\section{Introduction}

Pneumoconiosis is caused by coal dust being deposited in the lung, causing an inflammatory response and fibrosis in the lung tissue. Pneumoconiosis continues to progress even if exposure to dust is discontinued [1]. Therefore, the number of patients with pneumoconiosis continues to increase because the disease occurs 5-20 years after exposure to dust [1]. Furthermore, the incidence rate of pneumoconiosis among occupational diseases in South Korea is the highest [2]. Thus, care and management of pneumoconiosis are required, since the number of pneumoconiosis patients is continuously increasing.

Pneumoconiosis causes various mental problems as well as physical symptoms. Typical physical problems include dyspnea and various symptoms due to chronic respiratory distress

This paper was selected from the 2019 1st International Workshop on Human-centric Ideas, Policy and Advancement (HIPA-1 2019)

Article history:

Received (January 9, 2019), Review Result (February 9, 2019), Accepted (March 13, 2019) 
including cough, sputum, chest pain, and chronic fatigue [3]. In addition, most patients have mental health problems, such as depression and anxiety, because they know that pneumoconiosis is an incurable disease [4]. Therefore, interventions are needed to improve the mental health and physical health of pneumoconiosis patients, but until now there have been few intervention studies for patients with pneumoconiosis, and most have focused on physical health.

Recent studies in respiratory patients have shown that laughter therapy improved not only respiratory function but also depression and anxiety [5]. Therefore, this study aimed to determine the effect of the combined intervention of laughter and exercise on the physical and mental health of patients with pneumoconiosis.

\section{Methods}

\subsection{Study design, participants, and procedure}

The present study employed a quasi-experimental design using a nonequivalent control group pretest-post-test, non-synchronized design. The subjects were patients residing in a pneumoconiosis center in T city in the eastern part of South Korea.

A pre-test of patients who consented to participate in the study was conducted at the end of July 2015. After the pre-test, the exercise-only intervention was conducted for the comparison group, and a post-test was conducted after the intervention. Afterward, the combined intervention of laughter and exercise was conducted for the experimental group, followed by a post-test.

The participants engaged in a 50-minute intervention session three times a week for five weeks. In each session, the experimental group received a combined intervention of laughter and exercise (5-minute warm-up, 20-minute laughter treatment, 20-minute exercise treatment, and 5-minute wind down) while the comparison group was treated only with exercise intervention (10-minute warm-up, 30-minute exercise treatment, and 10-minute wind down).

\subsection{Data Collection and statistical analyses}

Data on sociodemographic variables, physical function, and mental health were collected before and after the intervention. Physical health-related variables included a ratio of the forced expiratory volume in the first second to the forced vital capacity of the lungs (an FEV1/FVC ratio), peripheral capillary oxygen saturation $\left(\mathrm{SPO}_{2}\right)$, and subjectively perceived dyspnea score. Mental health-related variables were depression, anxiety, and quality of life in patients with respiratory disease.

All data analyses were performed using IBM SPSS v.21.0. Differences in the baseline characteristics between the two groups were tested using a t-test and X2 test. A comparison of pre-and post-test data within each group was performed using paired t-tests. Lastly, differences between pre-and post-experimental data for each group were obtained, and differences between the experimental and comparison groups for each variable change were tested through independent t-tests.

\section{Results}

The baseline characteristics of the participants are presented in Table 1. No significant difference in baseline characteristics was found between the experimental and comparison groups. 
Table 1. Homogeneity test of the baseline characteristics between the experimental and the comparison groups

\begin{tabular}{|c|c|c|c|c|c|}
\hline Variables & Characteristics & $\begin{array}{c}\text { The experimental } \\
\text { group }(n=20) \\
N(\%) \text { or } \\
\text { mean } \pm \text { SD }\end{array}$ & $\begin{array}{c}\text { Comparison } \\
\text { group }(n=20) \\
N(\%) \text { or } \\
\text { mean } \pm \text { SD }\end{array}$ & $\begin{array}{l}\mathrm{X}^{2} \text { or } \mathrm{t}- \\
\text { value }\end{array}$ & $\begin{array}{c}p- \\
\text { value }\end{array}$ \\
\hline Age & - & $74.95 \pm 5.20$ & $76.60 \pm 5.27$ & 0.99 & 0.325 \\
\hline Height $(\mathrm{cm})$ & - & $163.70 \pm 6.61$ & $162.80 \pm 4.93$ & -0.49 & 0.628 \\
\hline Weight (kg) & - & $62.10 \pm 6.37$ & $64.49 \pm 8.23$ & 1.03 & 0.311 \\
\hline Duration of a career & - & $24.95 \pm 7.86$ & $25.05 \pm 10.38$ & 0.04 & 0.972 \\
\hline Years since & - & $15.37 \pm 8.90$ & $15.58 \pm 9.12$ & 0.07 & 0.943 \\
\hline \multirow{2}{*}{ Gender $^{\dagger}$} & Male & $19(95.0)$ & $19(95.0)$ & \multirow{2}{*}{-} & \multirow{2}{*}{1.000} \\
\hline & Female & $1(5.0)$ & $1(5.0)$ & & \\
\hline \multirow{3}{*}{ Education level $^{\dagger}$} & None & $9(45.0)$ & $6(30.0)$ & \multirow{3}{*}{ - } & \multirow{3}{*}{0.479} \\
\hline & Elementary school & $8(40.0)$ & $10(50.0)$ & & \\
\hline & Middle or high school & $3(15.0)$ & $4(20.0)$ & & \\
\hline \multirow{2}{*}{ Marital status ${ }^{\dagger \ddagger}$} & Married & $6(33.3)$ & $8(40.0)$ & \multirow[b]{2}{*}{ - } & \multirow{2}{*}{.820} \\
\hline & Separated/divorced & $12(66.7)$ & $12(60.0)$ & & \\
\hline \multirow{3}{*}{ Smoking } & Current smoker & $5(25.0)$ & $5(25.0)$ & \multirow{3}{*}{0.68} & \multirow{3}{*}{0.772} \\
\hline & Past-smoker & $12(60.0)$ & $10(50.0)$ & & \\
\hline & No- smoker & $3(15.0)$ & $5(25.0)$ & & \\
\hline \multirow{2}{*}{ Regular exercise } & Yes & $13(65.0)$ & $15(75.0)$ & \multirow{2}{*}{0.48} & \multirow{2}{*}{0.490} \\
\hline & No & $7(35.0)$ & $5(25.0)$ & & \\
\hline \multirow{3}{*}{ Physical health } & FEV1/FVC(\%) & $57.89(12.40)$ & $60.40(14.68)$ & 0.58 & 0.563 \\
\hline & $\mathrm{SPO}_{2}$ & $95.00(2.10)$ & $95.00(1.75)$ & 0.00 & 1.000 \\
\hline & Perceived dyspnea & $2.65(1.53)$ & $2.48(1.19)$ & -0.40 & 0.688 \\
\hline \multirow{3}{*}{$\begin{array}{l}\text { Psychological } \\
\text { health }\end{array}$} & Depression & $6.55(3.30)$ & $6.15(3.15)$ & -0.39 & 0.697 \\
\hline & Anxiety & $16.35(10.64)$ & $11.55(8.41)$ & -1.58 & 0.122 \\
\hline & Quality of life & $5.03(0.88)$ & $5.41(0.57)$ & 1.62 & 0.113 \\
\hline
\end{tabular}

Legend: $\uparrow$ Fisher's exact test; $\$ 2$ missing data in the experimental group

FEV1/FVC: a ratio of the forced expiratory volume in the first second to the forced vital capacity of the lungs

$\mathrm{SPO}$ : peripheral capillary oxygen saturation

[Table 2] shows differences in the within-group pre-and post-test scores of physical function and mental health variables of the experimental and comparison groups and a comparison of the magnitude of changes for each variable between the two groups. No significant difference was found between the experimental and comparison groups for the physical function-related variables, which are an FEV1/FVC ratio, $\mathrm{SPO}_{2}$, and subjectively perceived degree of dyspnea. Comparison of within-group pre-and post-test results showed a significant increase in $\mathrm{SPO}_{2}$ for the experimental group only but perceived dyspnea was significantly improved for both groups.

No significant between-group difference was found for the changes in depression, but the within-group comparison between pre-and post-test showed a significant decrease in depression scores of the experimental group. As a result of the comparison between the two groups, anxiety was significantly reduced in the experimental group. Quality of life was also significantly improved in the experimental group compared to the comparison group.

\section{Discussion}

In the case of physical health, no significant effect was found for an FEV1/FVC ratio, $\mathrm{SPO}_{2}$, and subjective perception of dyspnea between the experimental and comparison groups, but the results of the within-group pre- and post-test comparison showed a significant 
increase in $\mathrm{SPO}_{2}$ in the experimental group, and the improvement in the subjective perception of dyspnea was effective for both groups.

A comparison of physical changes between the present study and previous studies revealed the following. First, no significant difference in the intervention effect on an FEV1/FVC ratio was found between groups, and the within-group pre- and post-test differences were also nonsignificant, which is similar to the findings of previous studies [6][7]. This means that significant changes in an FEV1/FVC ratio through exercise intervention or combined intervention of laughter and exercise are not easy because pneumoconiosis is an irreversible change in the lungs.

Second, although there was no significant difference between the two groups in the case of $\mathrm{SPO}_{2}$, within-group pre- and post-test comparisons showed that a statistically significant increase was found only for the experimental group. This result reveals that there is a possibility that mild laughter may have helped to increase oxygen in the body because laughter pushes out more air left in the lungs, resulting in more oxygen being inhaled.

Third, even though subjectively perceived dyspnea was not significantly different between the two groups, within-group pre- and post-test comparisons showed significant differences for both groups. This is consistent with the findings of previous studies [6][7]. The finding does not mean there was no effect of the combined intervention of laughter and exercise on subjective dyspnea; rather, it means that both combined intervention of laughter and exercise and exercise-only intervention are effective for improving subjectively perceived dyspnea.

Table 2. Changes in physical and psychological health variables

\begin{tabular}{|c|c|c|c|c|c|c|c|c|c|c|c|c|}
\hline \multirow{2}{*}{ Variables } & \multicolumn{5}{|c|}{ The experimental group $(n=20)$} & \multicolumn{5}{|c|}{ Comparison group $(\mathrm{n}-20)$} & \multirow[t]{2}{*}{$t^{\dagger \dagger}$} & \multirow[t]{2}{*}{$p^{\dagger \dagger}$} \\
\hline & Pretest & Posttest & $\Delta$ (Post-Pre) & $\mathrm{t}^{\dagger}$ & $p^{\dagger}$ & Pretest & Posttest & $\Delta$ (Post-Pre) & $\mathrm{t}^{\dagger}$ & $p^{\dagger}$ & & \\
\hline FEV1/FVC \% & $57.89 \pm 12.40$ & $56.63 \pm 11.57$ & $1.26 \pm 7.43$ & 0.76 & .729 & $60.40 \pm 14.68$ & $62.14 \pm 10.09$ & $1.74 \pm 12 / 90$ & 0.60 & .277 & 0.90 & .687 \\
\hline $\mathrm{SPO}_{2}$ & $95.00 \pm 2.10$ & $96.60 \pm 1.35$ & $1.60 \pm 1.76$ & -4.07 & $<.001$ & $95.00 \pm 1.75$ & $95.55 \pm 2.16$ & $0.55 \pm 2.40$ & -1.03 & .159 & -1.58 & .061 \\
\hline Perceived dyspnea & $2.65 \pm 1.53$ & $1.38 \pm 0.99$ & $1.27 \pm 1.07$ & 5.33 & $<.001$ & $2.48 \pm 1.19$ & $1.15 \pm 1.11$ & $1.33 \pm 0.71$ & 8.32 & $<.001$ & 1.74 & .932 \\
\hline Depression & $6.55 \pm 3.30$ & $4.85 \pm 3.51$ & $1.70 \pm 4.09$ & 1.86 & .040 & $6.15 \pm 3.15$ & $5.10 \pm 3.01$ & $1.05 \pm 2.40$ & 1.96 & .033 & 0.61 & .272 \\
\hline Anxiety & $15.35 \pm 10.64$ & $7.85 \pm 6.84$ & $8.50 \pm 10.64$ & 3.57 & .001 & $11.55 \pm 8.41$ & $9.10 \pm 7.46$ & $2.45 \pm 7.46$ & 1.47 & .079 & 2.08 & .023 \\
\hline Quality of life & $5.03 \pm 0.88$ & $6.02 \pm 0.60$ & $0.99 \pm 0.88$ & -5.00 & $<.001$ & $5.41 \pm 0.57$ & $5.66 \pm 0.83$ & $0.25 \pm 0.71$ & -1.57 & .067 & -2.91 & .003 \\
\hline \multicolumn{13}{|c|}{ Legend: $+t$ and $p$ from paired $t$-test; $+\dagger t$ and $p$ from independent group $t$-test [comparison of $\Delta$ (Post-Pre) between the two } \\
\hline
\end{tabular}

As for mental health, the results of the present study showed significant effects on anxiety and the quality of life of patients with pneumoconiosis, which are consistent with previous studies [8]. It seems that the combined intervention of laughter and exercise that brings pleasant feelings reduced anxiety. In the previous study, the quality of life effectively improved in the 8 weeks or longer exercise program [9], but in this study, the combined intervention of laughter and exercise is a highly effective program that significantly improved the quality of life even though the intervention lasted for a relatively short period of five 
weeks. Lastly, pre-and post-test comparisons in both groups showed a significant decrease in depression. This means that both the combined intervention of laughter and exercise and the exercise-only intervention are effective in decreasing depression, which is consistent with previous studies [5][8].

\section{Conclusion}

Based on the findings of this study, the following conclusions were derived. While the results of the present study showed a partial effect of the combined intervention of laughter and exercise on the physical health of the patients with pneumoconiosis compared to the intervention of exercise only, the former was very effective in improving mental health.

\section{References}

[1] B. S. Choi, S. Y. Park, and J. O. Lee, "Current status of pneumoconiosis patients in Korea," Journal of Korean Medical Science, vol.25, pp.S13-S19, (2010) DOI: 10.3346/jkms.2010.25.S.S13

[2] S. K. Kang and E. A. Kim, "Occupational disease in Korea," Journal of Korean Medical Science, vol.25, pp.S4-S12, (2010)

[3] T. T. Bauer, C. M. Heyer, H. W. Duchna, K. Andreas, A. Weber, E. V. Schmidt, W. Ammenwerth, and G. Schultze-Werninghaus, "Radiological findings, pulmonary function and dyspnea in underground coal mines," Respiration, vol.74, pp.80-87, (2007) DOI: 10.1159/000090200

[4] J. Jang, Y. H. Lee, and M. K. Choi, "Cardiopulmonary symptoms, quality of sleep, and depression in the hospitalized patient with Pneumoconiosis,” Korean Journal of Adult Nursing, vol.23, no.2, pp.135-145, (2011)

[5] M. Y. Chang, "Effects of laughter therapy on depression in elderly," Journal of Digital Convergence, vol.12, no.6, pp.501-508, (2014) DOI: 10.14400/JDC.2014.12.6.501

[6] J. M. Lee, I. K. Park, J. K. Kim, G. J. Jeon, J. R. Kim, J. H. Kim, H. Cheong, and B. Y. Choi, "The clinical efficacy of an individualized pulmonary rehabilitation program in patients with coal-worker Pneumoconiosis," Korean Journal of Medicine, vol.87, No.6, pp.690-697, (2014)

[7] S. Y. Ryu, C. B. Park, J. K. Lim, H. Lee, H. J. Yu, and K. H. Cho, "Short-term inpatient pulmonary rehabilitation for pneumoconiosis," Journal of Korean Academy of Rehabilitation Medicine, vol.22, no.3, pp.705-710

[8] K. R. Lebowitz, S. Suh, P. T. Diaz, and C. F. Emery, "Effects of humor and laughter on psychological functioning, quality of life, health status, and pulmonary functioning among patients with chronic obstructive pulmonary disease: A preliminary investigation," Heart \& Lung, vol.40, no.4, pp.310-319, (2011) DOI: 10.1016/j.hrtlng.2010.07.010

[9] P. A. Pirraglia, B. Casserly, R. Velasco, M. L. Borgia, and L. Nici, L. "Association of change in depression and anxiety symptoms with functional outcomes in pulmonary rehabilitation patients," Journal of Psychosomatic Research, vol.71, no.1, pp.45-49, (2011) DOI: 10.1016/j.jpsychores.2011.01.002 
This page is empty by intention. 